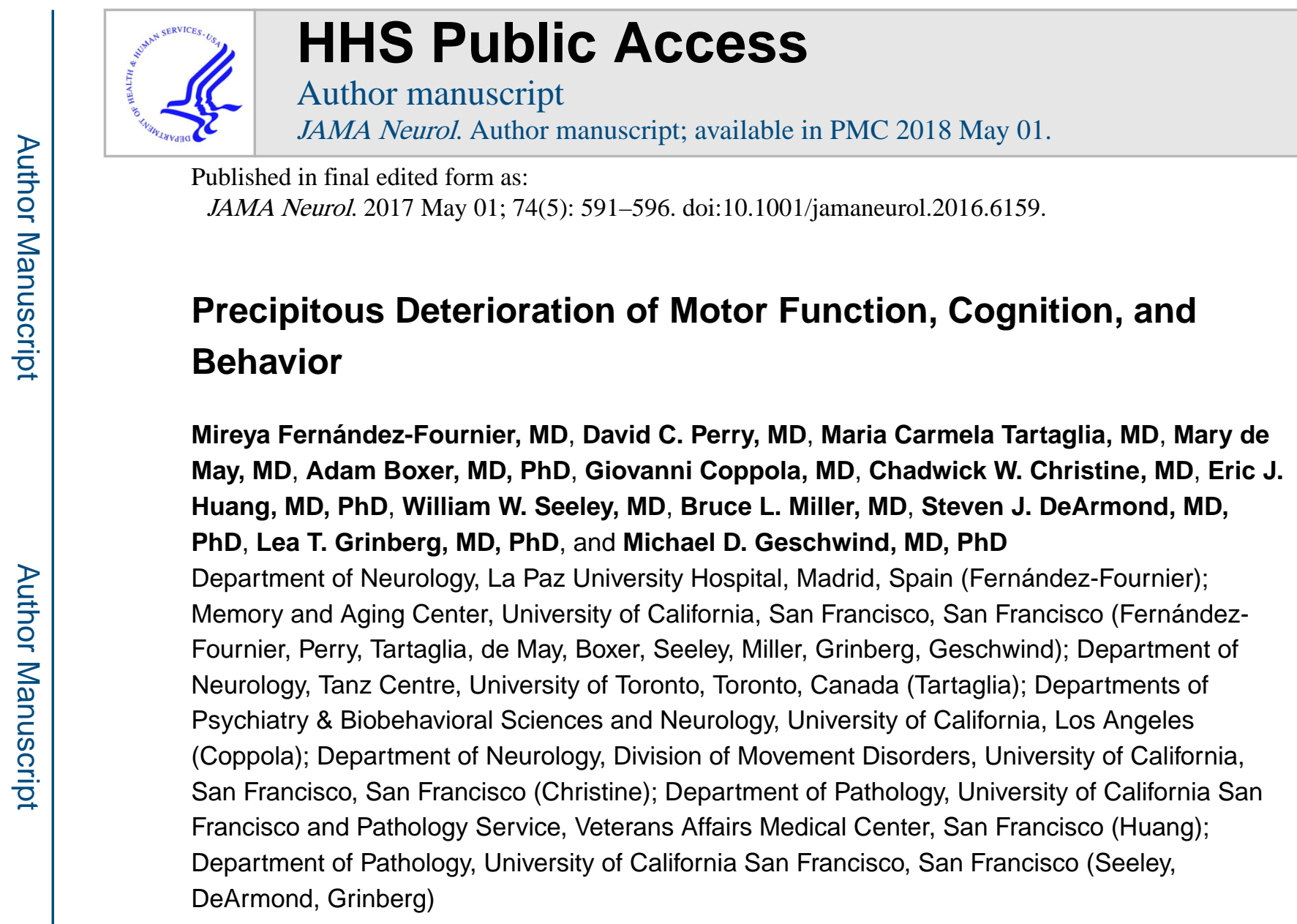

\begin{abstract}
A 72-year-old woman developed new-onset depression, sustained an unexplained fall, and started walking cautiously. After 1 year, her depression resolved but she developed a dry cough. One year later, she experienced a more rapid decline in her gait with parkinsonism, visual difficulties with restricted vertical gaze, slowed horizontal and vertical saccades, dysphagia, apathy, and progressive cognitive decline, which led to her death 2 years later. The differential diagnosis, neuroimaging, and pathological findings are discussed, as well as their public health implications.
\end{abstract}

\title{
Report of a Case
}

Following her son's death, a 72-year-old woman with a history of hypertension and hypothyroidism developed a year of severe depression, fell and broke her arm, and walked cautiously while dragging her right leg. A year later, she developed an unexplained dry

Corresponding Author: Michael D. Geschwind, MD, PhD, Memory and Aging Center, University of California, San Francisco, 675 Nelson Rising Ln, San Francisco, CA 94158 (michael.geschwind@ ucsf.edu).

Author Contributions: Drs Grinberg and Geschwind contributed equally to this article.

Role of the Funder/Sponsor: The National Institutes of Health, the Michael J. Homer Family Fund, and the Tau Consortium had no role in the design and conduct of the study; collection, management, analysis, or interpretation of the data; preparation, review, or approval of the manuscript; and decision to submit the manuscript for publication.

Additional Contributions: We thank Perluigi Gambetti, MD, PhD, US National Prion Disease National Pathology Surveillance Center, for his center's assistance with the pathological analysis; Wei Chen, BSc, US National Prion Disease Pathology Surveillance Center, who performed the Western blot; and Ignazio Cali, $\mathrm{PhD}$, Case Western Reserve University, for his help with the manuscript and figure labeling. None of these individuals were compensated for their contributions. We thank the patient's daughter for granting permission to publish this information. 
cough and hoarseness. Two years after her initial fall, she complained of weakness and developed gait instability, needing to hold on to something when walking ("onset"). From this point, her deterioration was quick and included a sense of leg weakness, "not seeing well," and pronounced apathy. Seven months after "onset," an examination revealed symmetrical hyperreflexia with a jaw jerk and an overactive gag reflex but was otherwise normal, failing to identify weakness or gait unsteadiness. Between months 7 and 13, she was dizzy when turning without experiencing vertigo, and began losing her balance, falling twice. At 14 months, she needed a cane and could not concentrate or manage her checkbook, but she was oriented to the date, had a knowledge of recent events, and had a 3 of 3 oneminute recall.

During months 14 through 16 she became more apathetic and depressed, had intermittent memory and orientation problems, worsening parkinsonism (bradykinesia, difficulty initiating movement, micrographia, emerging tremor, and intermittently needing a wheelchair) without ataxia, and favored her left leg when walking. By month 17 her apathy increased, and she occasionally dragged her right foot. On examination, she was alert and oriented to the month and year, had intact naming, and a normal cranial nerve examination, but she was intermittently tearful, had impaired spontaneous word generation and recall, mild hypomimia, mildly slowed bilateral rapid movements, retropulsion on standing, collapse on sitting, and normal stride only with assistance. She did, however, have normal strength, tone, sensation, reflexes, and plantar flexor responses without dysmetria.

At 19 months, she had dysphagia, dysarthria, word-finding problems, difficulty turning in bed, and leg movements and talking during sleep. Carbidopa-levodopa failed to improve symptoms. A neurological examination also revealed a blunted affect, a 10 of 25 Montreal Cognitive Assessment score, mild hypomimia, right gaze diplopia, an impaired upgaze, moderately slowed rapid alternating movements, a bilateral pronator drift, extreme micrographia, and generalized parkinsonism, requiring assistance to rise from a chair and walk.

At her first visit to the University of California, San Francisco Memory and Aging Center at 21 months, her family reported her intermittent word comprehension difficulty, paraphasias, pseudobulbar affect, and progressive myoclonus. Her examination showed distractibility, severe hypomimia, moderate hypophonia, slowed eyelid opening, a restricted vertical gaze, slowed horizontal and vertical saccades, a saccadic horizontal pursuit, marked axial and limb rigidity, extreme and predominantly right-sided bradykinesia, postural tremor, myoclonus, a marked grasp reflex, brisk reflexes, and upgoing plantar responses. Cognitive testing results revealed a Mini-Mental State Examination score of 7 of 30, severe memory impairment, an inability to copy a complex (the Benson) figure, and very impaired frontal-executive function and working memory. By 27 months, she had declined dramatically, experiencing sleepiness, intermittent eye opening, an inability to follow simple commands, mutism, and flexed, contracted, and spastic extremities. She died 28 months after "onset," with a possible disease course of more than 4 years. 


\section{Laboratory and Neuroradiological Data}

Extensive laboratory testing for dementia, including ceruloplasmin and serum/urine copper, yielded normal results. The patient's traumatic lumbar puncture at 27 months showed a mildly elevated protein level (white blood cell count, 1; red blood cell count, 985; protein, $53 \mathrm{mg} / \mathrm{dL}$ [normal = 0-50 mg/dL]; glucose, $63 \mathrm{mg} / \mathrm{dL}$ ).

Magnetic resonance imaging (MRI) at month 14 (Figure 1A and C) reported mild atrophy and "mild altered signal intensities in sublenticular white matter and basal ganglia, including caudate head, minutely increased on T1- and T2-weighted sequences," which "may reflect underlying pigment/mineral deposition." Magnetic resonance imaging at month 20 (Figure $1 \mathrm{~B}$ and $\mathrm{D}$ ) reported mild atrophy and mild small vessel disease. There was no change in the slight bilateral striatal T2-weighted hyperintensity.

\section{Clinical Discussion (Dr Perry)}

This patient's clinical course is characterized by an uncertain time of onset, with 2 years of nonprogressive, potentially related symptoms, including depression, a single fall, and dry cough with hoarseness. This was followed by a progressive neurological decline over 2 years involving multiple systems, with evidence of extrapyramidal, pyramidal, oculomotor, cognitive, and behavioral features. In evaluating patients who present late in their course with the involvement of multiple neurological systems and significant impairment, it is helpful to revisit the first symptoms, as neurodegenerative diseases often begin preferentially in a small number of regions. With the addition of more symptoms and an understanding of the progression rate, the clinician attempts to recognize known syndromes and looks for individual features with sufficient specificity to narrow the diagnostic possibilities. This patient's first symptoms were nonspecific. Depression can be the first manifestation of many neurodegenerative diseases. The presence of extrapyramidal, myoclonic, cognitive, and behavioral features suggests a more limited set of possibilities, with dementia with Lewy bodies being the most common. She had features, however, that made this unlikely, including eye movement abnormalities and early falls, which may indicate progressive supranuclear palsy (PSP). She met the diagnostic inclusion criteria for probable PSP, including an age of 40 years or older at onset, progressive disorder with early vertical supranuclear gaze palsy, and prominent postural instability with falls in the first year of onset. She also met supportive criteria, including symmetric akinesia, levodopa nonresponsive parkinsonism, early cognitive impairment, apathy, and dysphagia. ${ }^{1}$ The MRI striatal hyperintensity, downplayed by the radiological interpretation, however, might qualify as a relevant basal ganglia "structural" abnormality, which is an exclusion criterion for a PSP diagnosis. ${ }^{1}$ In addition, some of the patient's other features were atypical for PSP, including myoclonus, asymmetry, and no axial rigidity. Her disease duration was shorter than the average 5 to 7 years seen in PSP, although more rapid cases have been described. ${ }^{2}$

Corticobasal degeneration could also explain the patient's atypical parkinsonism, lack of levodopa responsiveness, myoclonus, and cognitive and behavioral features, but it would not be expected to cause her oculomotor findings until a late stage. Saccadic eye movement abnormalities have also been described in motor neuron disease (she had no lower motor 
neuron findings), multiple system atrophy, and spinocerebellar ataxia (she had no family history or cerebellar findings). A PSP syndrome has been described with some DCTN1 mutations, but again, she lacked a relevant family history. Vertical supranuclear gaze palsy, movement disorders, and cognitive and psychiatric symptoms are seen in late-onset Niemann-Pick $\mathrm{C}$ disease, though what is considered late for that illness would still be much earlier than this patient's age at onset. Wilson disease rarely occurs after age 70 years and can cause vertical eye movement abnormalities and parkinsonism, but she had negative copper and ceruloplasmin test results, making this less likely. Whipple disease can cause parkinsonism and vertical ophthalmoplegia, and though she had no gastrointestinal symptoms, a minority of cases have isolated central nervous system involvement.

Her MRI most strongly raises concern for prion disease, with striatal and cingulate hyperintensities more prominent on diffusion-weighted-imaging than on fluid-attenuated inversion recovery. ${ }^{3}$ Restricted diffusion in the striatum and cortex (cortical ribboning) on diffusion-weighted imaging is among the most sensitive and specific antemortem tests for detecting Jakob-Creutzfeldt Disease (JCD) with a diagnostic accuracy of $97 \% .{ }^{3}$ A 4-year time course would be longer than expected for JCD, and vertical gaze palsy with slowed saccadic velocity is not typically described. Prion disease, however, can present in numerous ways $^{4}$ and would explain her relatively rapid progression, myoclonus, parkinsonism, and cognitive impairment.

\section{Neuropathological Discussion (Drs Grinberg and DeArmond)}

A brain pathological assessment was performed at the University of California, San Francisco/Memory and Aging Center Neurodegenerative Disease Brain Bank and the National Prion Disease Pathology Surveillance Center. Postmortem delay was 12 hours and the brain weighed $1079 \mathrm{~g}$. A gross examination revealed general atrophy and ventriculomegaly with substantia nigra pigment loss, mildly thinned cortical ribbon, and a normal size midbrain. Microscopy showed extensive cortical vacuolation prominent in the deep layers, with colocalized astrogliosis and neuronal loss. Vacuolation also involved striatum and dentate nucleus of the cerebellum, but was mild in the hippocampus and was not present in the occipital cortex and cerebellar molecular layer. The substantia nigra showed a moderate loss of neuromelanin. $\beta$-Amyloid and phospho-tau immunostaining revealed low Alzheimer disease neuropathologic changes (National Institute on AgingAlzheimer's Association A1B1C0). Transactive response deoxyribonucleic acid binding protein- 43 (ie, TDP-43) and fused in sarcoma (ie, FUS) staining were negative.

Immunostaining using the anti-prion protein $(\mathrm{PrP})$ antibody $3 \mathrm{~F} 4$ showed diffuse or "synaptic" $\mathrm{PrP}^{\mathrm{Sc}}$ in most brain regions and no plaques. Molecular and genetic analyses showed type 1 prion, no mutation in the prion protein gene, and codon 129 polymorphism methionine-valine (ie, sJCDMV1) subtype ${ }^{5}$ but with atypical predominance of unglycosylated PK-resistant PrPSc (US National Prion Disease Pathology Surveillance Center) (Figure 2). Interestingly, her cerebrospinal fluid research test results, which returned post mortem, revealed normal total and phospho-tau levels, but novel biomarkers (Nterminal and central epitopes of the tau protein [BT2 + HT7; HT7 + Tau5]) as well as the 
neurofilament light chain were 9 to 19 times higher than typical forPSP, ${ }^{6,7}$ which additionally would have suggested against a PSP diagnosis.

\section{Conclusions}

Although classical sporadic JCD (sJCD) involves rapid dementia with ataxia and myoclonus, sJCD has a variable semiology and duration ${ }^{3}$ and is commonly misdiagnosed. ${ }^{4} \mathrm{~A}$ recent study showed that sJCD patients had almost 4 misdiagnoses before correctly being diagnosed, including atypical parkinsonian syndromes (4\%), but none with PSP. ${ }^{4}$ Although the initial criteria for "probable PSP" were reported to have a sensitivity of $83 \%$ and specificity of $100 \%$, both seem lower in clinical practice. ${ }^{8}$ In the literature, 12 prion cases mimicking PSP have been previously reported, with 5 having a long duration (29-53 months). To our knowledge, this is the first case of a patient with sJCD MV1 fulfilling probable PSP criteria. ${ }^{1}$

The median life expectancy for patients is about 5 months in the sJCDMV1 subtype, but with a wide range (1-54 months). ${ }^{9}$ Most other sJCD subtypes have median durations of about 1 year, with similarly large ranges. ${ }^{9}$ In this patient, it was difficult to determine the onset of symptoms because she was relatively stable for 2 years after her first fall, but her disease duration was at least 28 months from the onset of progressive neurological decline, which is within the range reported previously. ${ }^{9}$

Besides walking difficulty and instability, eye movement abnormalities can also lead to misdiagnosing JCD as PSP. Unfortunately, most prion disease cohort studies report oculomotor and visual symptoms as a combined category, making it difficult to study the true frequency of each. Oculomotor problems in SJCD and genetic prion disease have been reported, however, in case reports. ${ }^{10}$ In this case, early vision complaints but late oculomotor problems could be consistent with JCD. ${ }^{10}$ The late oculomotor problems were not as consistent with PSP, however. ${ }^{1,8}$

Ancillary test results might have been useful to diagnose JCD. The patient's 14-month sagittal MRIs suggested rostral midbrain atrophy, a signal sometimes helpful in differentiating PSP from PD or multiple system atrophy, ${ }^{11}$ but the striatal and subtle diffusion-weighted imaging cortical ribboning hyperintensities should have raised suspicion for JCD. Unfortunately, sJCD MRIs are often misread. ${ }^{3}$ Cerebrospinal fluid proteins (14-3-3, neuron-specific enolase, and total-tau) have moderate diagnostic utility, although their absence does not exclude JCD. ${ }^{3}$ An electroencephalogram, particularly in later stages, might have revealed periodic sharp wave complexes suggestive of JCD. ${ }^{3}$ In retrospect, she met World Health Organization 1998 clinical criteria for possible sJCD, which require symptoms but not ancillary tests (required for probable SJCD), which were never performed. ${ }^{3}$ She met European 2009 and University of California, San Francisco criteria ${ }^{3}$ for probable sJCD, due to her abnormal diffusion-weighted imaging MRI.

A neuropathological examination showed JCD-like features atypical for the SJCD MV1 subtype, in which vacuolation and $\mathrm{PrP}^{\mathrm{Sc}}$ deposition usually involves all cerebral cortex layers plus neostriatum, thalamus, and cerebellum, sparing hippocampus and brainstem 
nuclei. ${ }^{5}$ In this case, vacuolation showed a laminar pattern affecting mostly the inner cortical layers. Moreover, the midbrain was severely involved and the molecular layer of the cerebellum and occipital cortex were spared, unlike sJCDMV1. The substantia nigra involvement explains the profound parkinsonism. Some of the histopathological features of this case are reminiscent of SJCD MV2 and VV2 subtypes, although $\operatorname{PrP}^{\mathrm{Sc}}$ plaques are missing. ${ }^{5}$ A Western blot showed $\mathrm{PrP}^{\mathrm{Sc}}$ type 1 , but with an atypical ratio of di, mono, and unglycosylated $\operatorname{PrP}^{\mathrm{Sc}}$ isoforms that was at variance with sJCDMV1 subtype (as well as other sJCD subtypes) $)^{5}$ and resembling an atypical $\mathrm{PrPSc}^{\mathrm{Sc}}$ prior case report. ${ }^{12}$

Sporadic JCD, even MV1 subtypes, can have clinically atypical presentations, including PSP-like presentations, with a disease duration of more than 2 years. In this case, the presence of myoclonus, asymmetric features, a relatively rapid course, and the MRI findings should have suggested against PSP and raised suspicion for JCD. Had her MRI been interpreted properly, she might have been diagnosed correctly ante mortem. A careful read of a diffusion-weighted imaging/apparent diffusion coefficient MRI is essential in cases of atypical neurodegenerative diseases, particularly with unusually rapid presentations. In this case, cerebrospinal fluid was distributed to several research centers, and most disturbingly, the neuropathology laboratory personnel and equipment were unnecessarily exposed to $\mathrm{PrP}^{\mathrm{Sc}}$. An early and accurate diagnosis of sJCD is extremely important for infection control and prognostic reasons.

\section{Acknowledgments}

Funding/Support: This article was supported by NIH/NIA R01AG031189, P01 AG019724, P50AG023501, the Michael J. Homer Family Fund, and the Tau Consortium.

\section{Conflict of Interest Disclosures}

Dr Perry is supported by grant K23AG045289 from the National Institute on Aging (NIA). Dr Boxer receives research support from grants R01AG038791 and U54NS092089 from the National Institutes of Health (NIH), the University of California, the Tau Consortium, CBD Solutions, the Bluefield Project to Cure FTD, the Alzheimer's Association, and the following companies: Avid, Biogen, Bristol Myers Squibb, C2N Diagnostics, Cortice Biosciences, Eli Lilly, Forum Pharmaceuticals, Genentech, Roche and TauRx. Dr Boxer has served as a consultant for Abbvie, Asceneuron, Ipierian, Ionis Pharmaceuticals, Janssen, and Merck; he serves on a Data and Safety Monitoring Board for Neurogenetics Pharmaceuticals; and he has stock and/or options in Alector and Delos. Dr Coppola is supported by grants RC1AG035610 and R01MH097268 from the NIH, the Tau Consortium, and the The John Douglas French Alzheimer's Foundation. Dr Miller receives grant support from the NIH/NIA and the Centers for Medicare \& Medicaid Services as grants for the Memory and Aging Center. He serves as the Medical Director for the John Douglas French Foundation; Scientific Director for the Tau Consortium; Director/Medical Advisory Board of the Larry L. Hillblom Foundation; and Scientific Advisory Board Member for the National Institute for Health Research Cambridge Biomedical Research Centre and its subunit, the Biomedical Research Unit in Dementia. Dr Grinberg is supported by grants R01AG040311 and K24AG053435 from the NIH, institutional grants P50AG023501 and P01AG019724 from the University of California, San Francisco, and grant support from the 
Tau Consortium and AVID Radiopharmaceuticals. Dr Geschwind serves on the board of directors for San Francisco Bay Area Physicians for Social Responsibility and on the editorial board of Dementia \& Neuropsychologia, and serves or has served as a consultant for Advanced Medical, Inc, Best Doctors, Grand Rounds Inc, Biohaven Pharmaceuticals Inc, Gerson Lehrman Group, Inc, Guidepoint Global, LLC, Lewis Brisbois Bisgaard \& Smith LLP, Kendall Brill \& Kelly LLP, MEDACorp, Franciscan Physician Network, Teva Pharmaceuticals, LCN Consulting, and Quest Diagnostics, Optio Biopharma Solutions. He receives support from grant R01 AG AG031189 from the NIA, the Michael J. Homer Family Fund, Quest Diagnostics, CurePSP, and the Tau Consortium. No other disclosures were reported.

\section{References}

1. Litvan I, Agid Y, Calne D, et al. Clinical research criteria for the diagnosis of progressive supranuclear palsy (Steele-Richardson-Olszewski syndrome): report of the NINDS-SPSP international workshop. Neurology. 1996; 47(1):1-9. [PubMed: 8710059]

2. Armstrong MJ, Castellani RJ, Reich SG. "Rapidly" progressive supranuclear palsy. Movement Disord Clin Pract. 2014; 1(1):70-72. dx.doi.org/10.1002/mdc3.12011.

3. Geschwind MD. Prion Diseases. Continuum (Minneap Minn). 2015; 21(6 Neuroinfectious Disease): 1612-1638. [PubMed: 26633779]

4. Paterson RW, Torres-Chae CC, Kuo AL, et al. Differential diagnosis of Jakob-Creutzfeldt disease. Arch Neurol. 2012; 69(12):1578-1582. [PubMed: 23229042]

5. Parchi P, Giese A, Capellari S, et al. Classification of sporadic Creutzfeldt-Jakob disease based on molecular and phenotypic analysis of 300 subjects. Ann Neurol. 1999; 46(2):224-233. [PubMed: 10443888]

6. Scherling CS, Hall T, Berisha F, et al. Cerebrospinal fluid neurofilament concentration reflects disease severity in frontotemporal degeneration. Ann Neurol. 2014; 75(1):116-126. [PubMed: 24242746]

7. Wagshal D, Sankaranarayanan S, Guss V, et al. Divergent CSF $\tau$ alterations in two common tauopathies: Alzheimer's disease and progressive supranuclear palsy. J Neurol Neurosurg Psychiatry. 2015; 86(3):244-250. [PubMed: 24899730]

8. Williams DR, Lees AJ. Progressive supranuclear palsy: clinicopathological concepts and diagnostic challenges. Lancet Neurol. 2009; 8(3):270-279. [PubMed: 19233037]

9. Collins SJ, Sanchez-Juan P, Masters CL, et al. Determinants of diagnostic investigation sensitivities across the clinical spectrum of sporadic Creutzfeldt-Jakob disease. Brain. 2006; 129(Pt 9):22782287. [PubMed: 16816392]

10. Armstrong RA. Creutzfeldt-Jakob disease and vision. Clin Exp Optom. 2006; 89(1):3-9. [PubMed: 16430434]

11. Graber JJ, Staudinger R. Teaching NeuroImages: "penguin" or "hummingbird" sign and midbrain atrophy in progressive supranuclear palsy. Neurology. 2009; 72(17):e81. [PubMed: 19398699]

12. Zanusso G, Polo A, Farinazzo A, et al. Novel prion protein conformation and glycotype in Creutzfeldt-Jakob disease. Arch Neurol. 2007; 64(4):595-599. [PubMed: 17420324] 

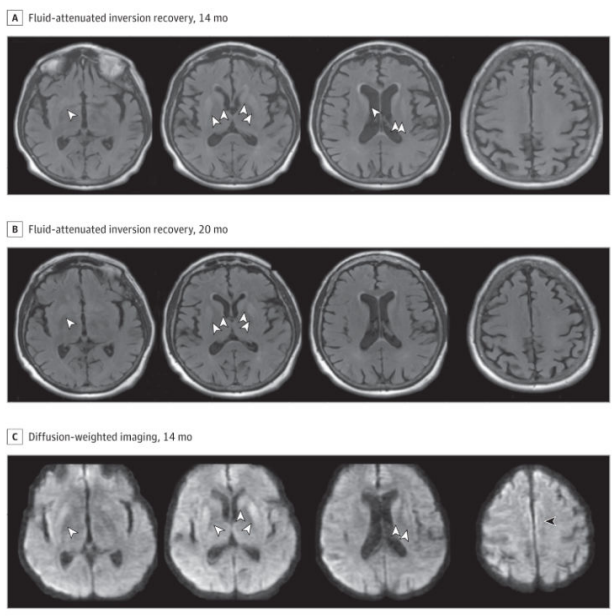

$D$ Diffusion-weighted imaging, $20 \mathrm{mo}$
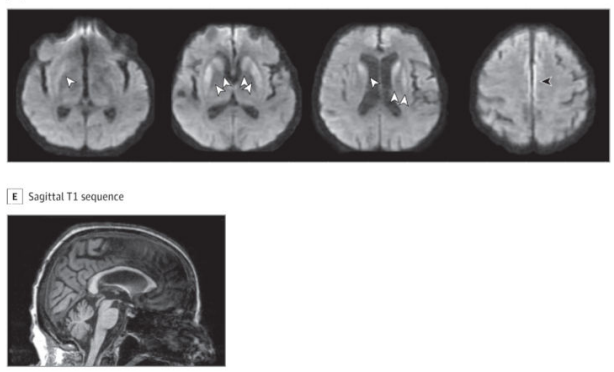

Figure 1. Brain Magnetic Resonance Imaging

Fluid-attenuated inversion recovery (A and B) and diffusion-weighted imaging ( $\mathrm{C}$ and $\mathrm{D})$ sequences at 14 (A and C) and 20 months (B and D), respectively, showing striatal (white arrowheads) and midcingulate cortical ribboning (black arrowhead) hyperintensities consistent with prion disease. Note that the abnormalities are identified more easily on diffusion-weighted imaging than fluid-attenuated inversion recovery sequences. The sagittal T1 sequence (E) shows a possible hummingbird sign with an atrophied midbrain compared with the pons, which some investigators feel is a helpful, distinguishing feature of progressive supranuclear palsy vs idiopathic Parkinson disease and other parkinsonian disorders. 


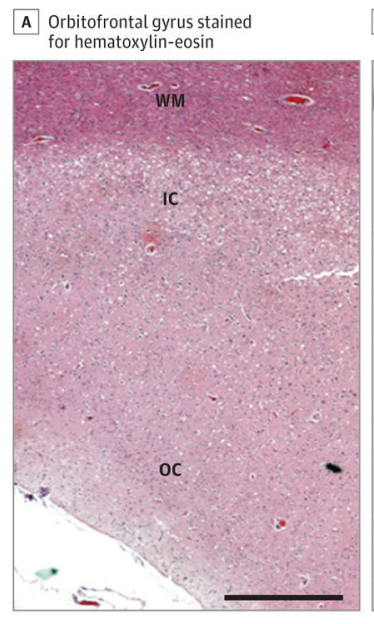

E Cerebellum at low magnification

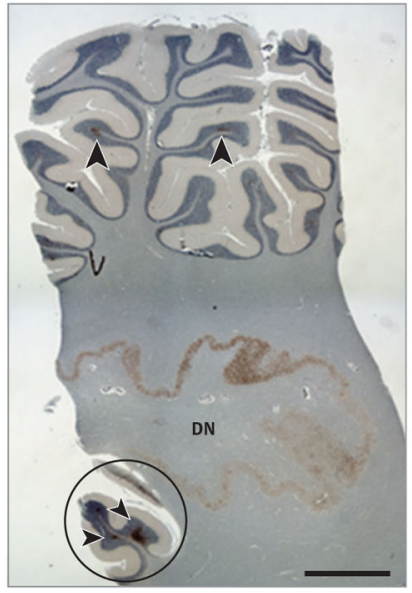

B Inferior frontal gyrus stained for prion protein

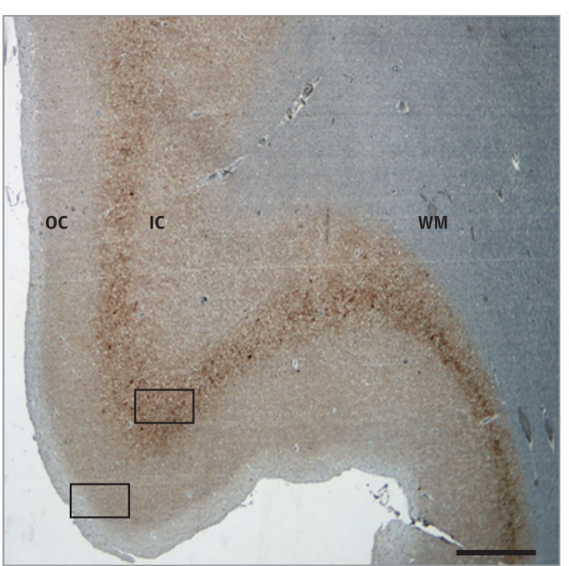

F Molecular layer of cerebellar hemispheres
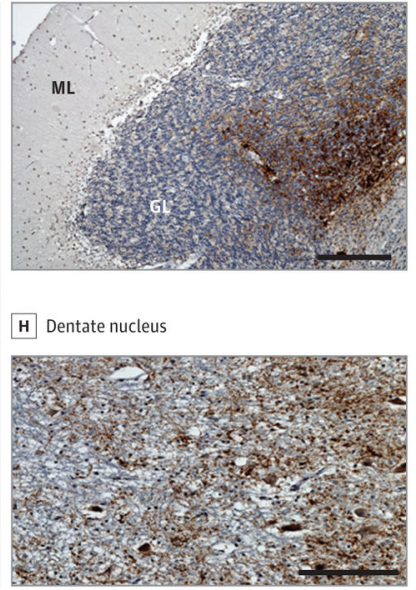

C Cerebral cortex stained for prion protein

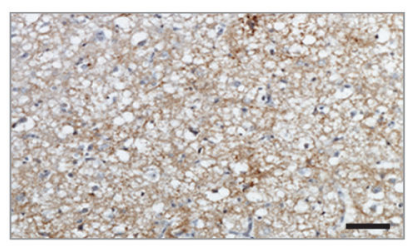

D Outer cortical layers stained for prion protein

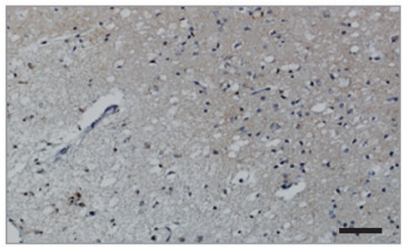

G Superior colliculi

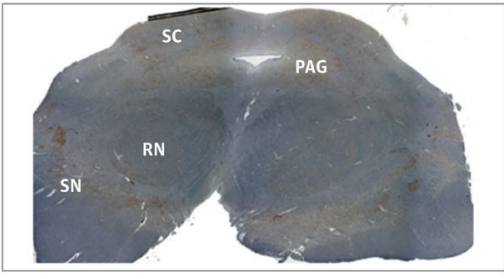

I Typing of prion protein by Western immunoblot

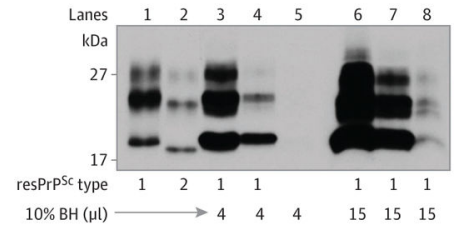

Figure 2. Pathological Findings: Histopathology and Prion-Related Protein Scrapie $\left(\right.$ PrPSc $^{\text {Sc }}$ Western Immunoblot

A, Orbitofrontal gyrus stained for hematoxylin-eosin. Vacuolation affects predominantly the inner cortical layers. B-H, Immunohistochemistry. B, Inferior frontal gyrus immunostained for $\operatorname{PrP}(3 \mathrm{~F} 4)$. In the cerebral cortex, $\mathrm{PrP}^{\mathrm{Sc}}$ had a laminar pattern of distribution and stained mainly layers IV-VI (brownish color), magnified in C, and relatively spared the outer cortical layers, magnified in $\mathrm{D}$ (black boxes in $\mathrm{B}$ show the regions magnified in $\mathrm{C}$ and $\mathrm{D}$ ). $\mathrm{E}$, Cerebellum at low magnification. Prion-related protein scrapie immunostaining with focal distribution (arrowheads) in the cerebellar granular layer is especially intense in the flocculus (circle) (magnified in F). The molecular layer of cerebellar hemispheres did not show $\mathrm{PrP}^{\mathrm{Sc}}$ immunostaining. Intense $\mathrm{PrP}^{\mathrm{Sc}}$ immunostaining was also seen in the dentate nucleus (magnified in $\mathrm{G}$ ) and in the brainstem (including tectum, substantia nigra, dorsal raphe nucleus, and nucleus of the solitary tract; not shown). H, Superior colliculi (tectum), periaqueductal gray, and substantia nigra of midbrain shows abundant $\mathrm{PrPSc}^{\mathrm{Sc}}$ deposition. I, Typing of $\mathrm{PrP}^{\mathrm{Sc}}$ by Western immunoblot. Brain homogenates (BHs) with antibody $3 \mathrm{~F} 4$ from the present case (lanes 3-8), as well as sCJDMM1 (lane 1) and sCJDMM2 (lane 2) control cases were subjected to digestion with $5 \mathrm{U} / \mathrm{mL}$ (corresponding to approximately $100 \mu \mathrm{g} / \mathrm{mL}$ ) proteinase $\mathrm{K}$ (PK). The unglycosylated (lowest band) PK-resistant $\mathrm{PrP}^{\mathrm{Sc}}\left(\mathrm{resPrP} \mathrm{Pc}^{\mathrm{Sc}}\right.$ ) from the 
frontal cortex (lanes 3 and 6), parietal cortex (lanes 4 and 7), and cerebellum (lanes 5 and 8) of the present case co-migrated at approximately $21 \mathrm{kDa}$ with unglycosylated resPrP $\mathrm{Pc}^{\mathrm{Sc}}$ type 1 associated with sCJDMM1 (lane 1) but not with the $\sim 19 \mathrm{kDa}$ unglycosylated resPrP ${ }^{\mathrm{Sc}}$ type 2 of sCJDMM2 (lane 2). Lanes 3 to 5 were rerun with less BH than lanes 6 to 8, and lanes 3 to 4 clearly show the predominance of unglycosylated $\operatorname{resPrP}^{\mathrm{Sc}}$, which is atypical for MV1. Scale bars: A, B, and E: $500 \mu \mathrm{m}$; C, D, and G: $50 \mu \mathrm{m}$; F: $100 \mu \mathrm{m}$; H: $1 \mathrm{~cm}$. DN indicates dentate nucleus; GL, granular layer; IC, inner cortical layers; ML, molecular layer; OC, outer cortical layers; PAG, periaqueductal gray; PrPSc, prions; SC, superior colliculus; SN, substantia nigra; u, unglycosylated; WM, white matter. 